\title{
Blind FIR Equalisation for High-Order QAM Signalling
}

\author{
S. Chen, T.B. Cook and L.C. Anderson \\ Department of Electronics and Computer Science \\ University of Southampton \\ Southampton SO17 1BJ \\ United Kingdom \\ E-mail: sqc@ecs.soton.ac.uk
}

\begin{abstract}
We compare some blind finite-impulse-response (FIR) equalisation schemes for high-order quadrature amplitude modulation (QAM) signalling. The popular constant modulus algorithm (CMA) is used as a benchmark in an investigation of two blind FIR equalisers, namely, a bootstrap maximum $a$ posteriori probability (MAP) equaliser and a recently introduced concurrent CMA and decision directed (DD) equaliser (CMA+DD). Both equalisers are known to outperform the CMA considerably at costs of small increase in computational complexity. Our study indicates that the bootstrap MAP equaliser has a faster convergence rate than the concurrent $\mathrm{CMA}+\mathrm{DD}$ equaliser.
\end{abstract}

\section{INTRODUCTION}

For communication systems employing high bandwidthefficiency QAM signalling, the CMA based FIR equaliser is a popular blind equalisation scheme [1],[2]. It has very simple computational requirements. A serious problem of the CMA is that it only achieves a moderate mean square error (MSE) after convergence, which may not be sufficient for the system to obtain adequate performance. A possible solution is to switch to a DD adaptation to minimise the CMA steady state MSE [3]. However, as pointed out in [4], in order for such a transfer to be successful, the CMA steady state MSE should be sufficiently low, which may not always be achievable in practice. In ICC 2001, De Castro et al [4] have suggested an interesting solution to this problem by operating a DD equaliser concurrently with a CMA equaliser. The weight adaptation of the DD equaliser follows that of the CMA equaliser, and the DD adjustment only takes place if the CMA is judged to achieve a successful adjustment. At a small cost of doubling complexity to that of the very simple CMA, this concurrent CMA+DD equaliser is reported to obtain a dramatical improvement in equalisation performance over the CMA [4].

The CMA and this newly introduced concurrent CMA+DD equaliser belong to a class of blind FIR equalisers commonly referred to as Bussgang algorithms [5]-[9]. In this study, we revisit a Bussgang blind equaliser called the bootstrap MAP equaliser, which was originally derived in [10] for 4-QAM constellation and extended to $M$-QAM $(M>4)$ channels in [8],[9]. We compare this bootstrap MAP equaliser with the concurrent CMA+DD equaliser, using the CMA as a benchmark in terms of complexity and performance. The bootstrap MAP equaliser requires similar numbers of multiplications and additions as the CMA, with an additional need of evaluating 4 exponential function values. Simulation confirms that both the bootstrap MAP and concurrent CMA+DD equalisers outperform the CMA considerably, and the results also suggest that the bootstrap MAP equaliser achieves faster convergence than the concurrent CMA+DD equaliser for the channel simulated.

\section{BLIND EQUALISATION}

Consider the baseband model of a digital communication channel characterized by a symbol-space FIR filter and an additive Gaussian white noise source. Specifically, the received signal at sample $k$ is given by

$$
r(k)=\sum_{i=0}^{n-1} a_{i} s(k-i)+e(k)
$$

where $n$ is the length of the channel impulse response (CIR), $a_{i}=a_{i R}+j a_{i I}$ are the channel taps, the symbol sequence $s(k)=s_{R}(k)+j s_{I}(k)$ is independently identically distributed, $e(k)=e_{R}(k)+j e_{I}(k)$ is a Gaussian white noise with $\mathrm{E}\left[e_{R}^{2}(k)\right]=\mathrm{E}\left[e_{I}^{2}(k)\right]=\sigma_{e}^{2}$, and $\mathrm{E}[\cdot]$ denotes the expectation. The symbol constellation is $M$-QAM and the set of all the symbol points is defined by

$$
\mathcal{S}=\left\{s_{i l}=(2 i-Q-1)+j(2 l-Q-1), 1 \leq i, l \leq Q\right\}
$$

where $Q=\sqrt{M}=2^{L}$, and $L$ is an integer.

A symbol-space equaliser is employed, which has an FIR structure defined by

$$
y(k)=\sum_{i=0}^{m-1} w_{i} r(k-i)=\mathbf{w}^{T} \mathbf{r}(k)
$$

where $m$ is the equaliser order, $\mathbf{w}(k)=\left[w_{0} w_{1} \cdots w_{m-1}\right]^{T}$ is the equaliser weight vector with $w_{i}=w_{i R}+j w_{i I}$, and $\mathbf{r}(k)=$ $[r(k) r(k-1) \cdots r(k-m+1)]^{T}$ the equaliser input vector. 
To deal with non-minimum phase channels, the equaliser has a delay approximately $k_{d} \approx m / 2$. Before blind adaptation, the equaliser weights are initialised to $w_{i}=1+j 0$ for $i=k_{d}$ and $w_{i}=0+j 0$ for $i \neq k_{d}$.

\section{A. The constant modulus algorithm}

The CMA adjusts the equaliser weights by minimising

$$
\bar{J}_{\mathrm{CMA}}(\mathbf{w})=\mathrm{E}\left[\left(|y(k)|^{2}-\Delta_{2}\right)^{2}\right]
$$

using a stochastic gradient algorithm, where $\Delta_{2}$ is a real positive constant defined by

$$
\Delta_{2}=\mathrm{E}\left[|s(k)|^{4}\right] / \mathrm{E}\left[|s(k)|^{2}\right] .
$$

At sample $k$, given $y(k)=\mathbf{w}^{T}(k) \mathbf{r}(k)$, the CMA adapts $\mathbf{w}$ according to [1], [2]

$$
\left.\begin{array}{l}
\epsilon(k)=y(k)\left(\Delta_{2}-|y(k)|^{2}\right) \\
\mathbf{w}(k+1)=\mathbf{w}(k)+\mu \epsilon(k) \mathbf{r}^{*}(k)
\end{array}\right\}
$$

where $\mu$ is a small positive adaptive gain and $\mathbf{r}^{*}(k)$ is the complex conjugate of $\mathbf{r}(k)$.

The computational complexity of the CMA is summarised in Table I. Let $\mathbf{w}_{\text {opt }}$ be the solution of the adaptive equaliser based on the cost function (4) that yields the correct signal constellation. All the weight vectors

$$
\mathbf{w}_{s}=\exp (j \phi) \mathbf{w}_{\mathrm{opt}}, \quad 0 \leq \phi<2 \pi,
$$

produces the same cost as $\bar{J}_{\mathrm{CMA}}\left(\mathbf{w}_{\mathrm{opt}}\right)$. Thus the CMA blind equaliser may converge to any of the solutions defined in (7). This undesired phase shift cannot be resolved by the CMA and must be eliminated by other means.

\section{B. The concurrent $C M A+D D$ equaliser}

De Castro et al [4] proposed a concurrent CMA and DD blind equalisation scheme. Specifically, let

$$
\mathbf{w}=\mathbf{w}_{c}+\mathbf{w}_{d}
$$

where $\mathbf{w}_{c}$ is designed to minimise the CMA cost function and $\mathbf{w}_{d}$ is designed to minimise the decision based MSE

$$
\bar{J}_{\mathrm{DD}}\left(\mathbf{w}_{d}\right)=\frac{1}{2} \mathrm{E}\left[|\mathcal{Q}[y(k)]-y(k)|^{2}\right]
$$

with $\mathcal{Q}[y(k)]$ denoting the quantized equalizer output

$$
\mathcal{Q}[y(k)]=\arg \min _{s_{i l} \in \mathcal{S}}\left|y(k)-s_{i l}\right|^{2} .
$$

TABLE I

COMPARISON OF COMPLEXITY PER WEIGHT UPDATE.

\begin{tabular}{cccc}
\hline equaliser & multiplications & additions & $\exp (\cdot)$ \\
\hline CMA & $8 \times m+6$ & $8 \times m$ & - \\
CMA+DD & $16 \times m+8$ & $20 \times m$ & - \\
MAP & $8 \times m+22$ & $8 \times m+16$ & 4 \\
\hline
\end{tabular}

At sample $k$, given $y(k)=\mathbf{w}_{c}^{T}(k) \mathbf{r}(k)+\mathbf{w}_{d}^{T}(k) \mathbf{r}(k)$, the CMA adapts $\mathbf{w}_{c}$ according to the rule (6) with an adaptive gain $\mu_{c}$. The DD adaptation follows immediately after the CMA adaptation and it only takes place if the CMA adjustment is viewed to be a successful one. Let

$$
\tilde{y}(k)=\mathbf{w}_{c}^{T}(k+1) \mathbf{r}(k)+\mathbf{w}_{d}^{T}(k) \mathbf{r}(k) .
$$

Then the DD part adjusts $\mathbf{w}_{d}$ according to [4]

$$
\begin{aligned}
\mathbf{w}_{d}(k+1) & =\mathbf{w}_{d}(k)+\mu_{d} \delta(\mathcal{Q}[\tilde{y}(k)]-\mathcal{Q}[y(k)]) \\
& \times(\mathcal{Q}[y(k)]-y(k)) \mathbf{r}^{*}(k)
\end{aligned}
$$

where $\mu_{d}$ is the adaptive gain of the DD equaliser and

$$
\delta(x)= \begin{cases}1, & x=0+j 0 \\ 0, & x \neq 0+j 0\end{cases}
$$

It is seen that $\mathbf{w}_{d}$ is updated only if the equaliser hard decisions before and after the CMA adaptation are the same.

The complexity of the CMA+DD algorithm, given in Table I, is linear in the equaliser order $m$. Let $\mathbf{w}_{d o p t}$ be the solution of the DD equaliser based on the cost function (9) that yields the correct signal constellation. The weight vectors that produces the same cost as $\bar{J}_{\mathrm{DD}}\left(\mathbf{w}_{d \mathrm{opt}}\right)$ are:

$$
\mathbf{w}_{d s}=\exp (j \phi) \mathbf{w}_{d \mathrm{opt}}, \quad \phi=0, \frac{\pi}{2}, \pi, \frac{3 \pi}{2} .
$$

The DD adaptation does not suffer from a serious phase shift problem, as the CMA does, and is capable of lowering the steady state MSE, compared with the CMA.

\section{The bootstrap MAP equaliser}

After the equalisation is accomplished, the equaliser output can approximately be expressed in two terms

$$
y(k) \approx x(k)+v(k)
$$

where $x(k)=s\left(k-k_{d}\right)$ and $v(k)=v_{R}(k)+j v_{I}(k)$ is approximately white Gaussian. That is, $y(k)$ can be modelled approximately by $M$ Gaussian clusters with means

$$
y_{i l}=s_{i l}, \quad 0 \leq i, l \leq Q,
$$

and all the clusters have an approximate covariance

$$
\left[\begin{array}{cc}
\mathrm{E}\left[v_{R}^{2}(k)\right] & \mathrm{E}\left[v_{R}(k) v_{I}(k)\right] \\
\mathrm{E}\left[v_{I}(k) v_{R}(k)\right] & \mathrm{E}\left[v_{I}^{2}(k)\right]
\end{array}\right] \approx\left[\begin{array}{cc}
\rho & 0 \\
0 & \rho
\end{array}\right] .
$$

Thus, the a posteriori p.d.f. of $y(k)$ is approximately

$$
p(\mathbf{w}, y(k)) \approx \sum_{q=1}^{Q} \sum_{l=1}^{Q} \frac{p_{q l}}{2 \pi \rho} \exp \left(-\frac{\left|y(k)-y_{q l}\right|^{2}}{2 \rho}\right)
$$

where $p_{q l}$ are the a priori probabilities of $y_{q l}$, and they are all 
equal. The bootstrap MAP equaliser is designed to maximise the a posteriori p.d.f. criterion

$$
\bar{\eta}(\mathbf{w})=\mathrm{E}[\eta(\mathbf{w}, y(k))]
$$

with

$$
\eta(\mathbf{w}, y(k))=\rho \sum_{q=1}^{Q} \sum_{l=1}^{Q} \exp \left(-\frac{\left|y(k)-y_{q l}\right|^{2}}{2 \rho}\right) .
$$

At sample $k$, given $y(k)=\mathbf{w}^{T}(k) \mathbf{r}(k)$, the equaliser weights can be adapted according to

$$
\mathbf{w}(k+1)=\mathbf{w}(k)+\mu \frac{\partial \eta(\mathbf{w}(k), y(k))}{\partial \mathbf{w}}
$$

with the adaptive gain $\mu$ and the stochastic gradient

$$
\begin{aligned}
\frac{\partial \eta(\mathbf{w}(k), y(k))}{\partial \mathbf{w}} & =\sum_{q=1}^{Q} \sum_{l=1}^{Q} \exp \left(-\frac{\left|y(k)-y_{q l}\right|^{2}}{2 \rho}\right) \\
& \times\left(y(k)-y_{q l}\right) \mathbf{r}^{*}(k) .
\end{aligned}
$$

In order to speed up convergence rate and to keep the complexity to a minimum, a multi-stage implementation was proposed [8],[9] for high-oder QAM signalling.

For the 16-QAM case, the equalisation objective is decomposed into a two-stage process. At the first stage, a 4cluster p.d.f. model is adopted with the cluster means being $\{ \pm 2+j \pm 2\}$. The equaliser weights are adjusted using this equivalent "4-QAM" model via the gradient algorithm (21). The objective of this stage is to achieve a roughly correct classification of equaliser outputs into the 4 quadrants in the complex plane. At the second stage, the 16-cluster p.d.f. model is adopted with the cluster means being the correct symbol points $\left\{s_{q l}, 1 \leq q, l \leq 4\right\}$. This cluster model is divided into 4 submodels, one for each quadrant. If the equaliser output is in a particular quadrant, the corresponding 4-cluster sub-model is used to adapt the equaliser weights via the gradient algorithm (21). As the adaptation at this stage is done correctly with high probability owing to the clustering of the previous stage, the overall equalisation objective can be achieved faster and more reliably.

TABLE II

A 22-TAP TELEPHONE CHANNEL IMPULSE RESPONSE FROM [11].

\begin{tabular}{cccccc}
\hline Tap & $\mathrm{Re}$ & $\mathrm{Im}$ & $\mathrm{Tap}$ & $\mathrm{Re}$ & $\mathrm{Im}$ \\
\hline 0 & 0.0145 & -0.0006 & 11 & 0.0294 & -0.0049 \\
1 & 0.0750 & 0.0176 & 12 & -0.0181 & 0.0032 \\
2 & 0.3951 & 0.0033 & 13 & 0.0091 & 0.0003 \\
3 & 0.7491 & -0.1718 & 14 & -0.0038 & -0.0023 \\
4 & 0.1951 & 0.0972 & 15 & 0.0019 & 0.0027 \\
5 & -0.2856 & 0.1896 & 16 & -0.0018 & -0.0014 \\
6 & 0.0575 & -0.2096 & 17 & 0.0006 & 0.0003 \\
7 & 0.0655 & 0.1139 & 18 & 0.0005 & 0.0000 \\
8 & -0.0825 & -0.0424 & 19 & -0.0008 & -0.0001 \\
9 & 0.0623 & 0.0085 & 20 & 0.0000 & -0.0002 \\
10 & -0.0438 & 0.0034 & 21 & 0.0001 & 0.0006 \\
\hline
\end{tabular}

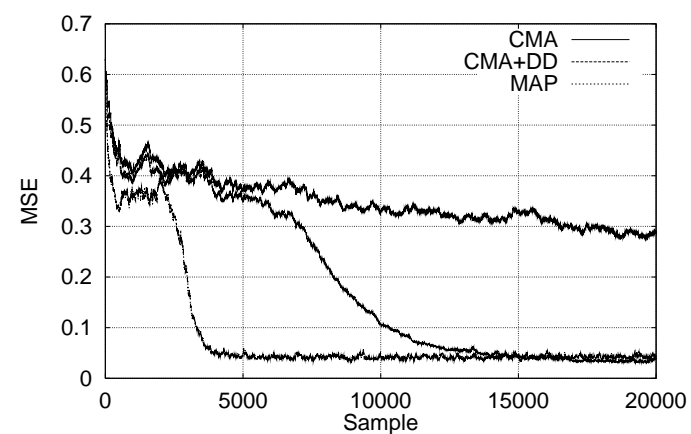

(a)

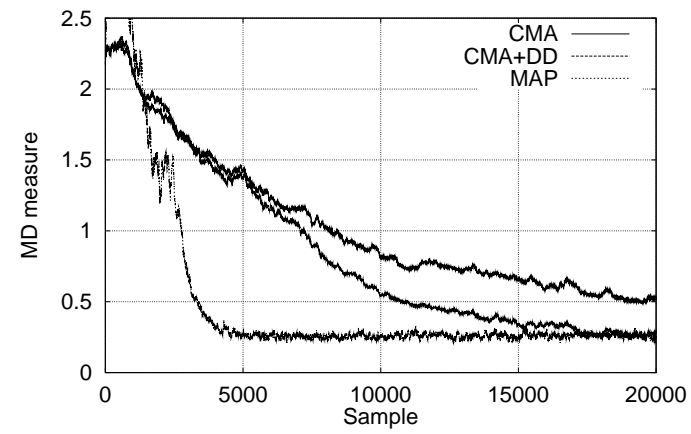

(b)

Fig. 1. Comparison of convergence performance in terms of (a) estimated MSE and (b) MD measure.

In general, the $M$-QAM equalisation, where $M=2^{2 L}$, can be achieved using the $L$-stage process. Because the sub-task of each stage can be accomplished easily and reliably, the overall convergence of the equaliser is achieved faster and more reliably. The soft-decision directed nature of this bootstrap MAP means that a much large adaptive gain can be used, which otherwise would cause the CMA to diverge. The choice of $\rho$ for each adaptation stage should ensure a proper separation of the clusters. If the value of $\rho$ is too large, a desired degree of separation among the clusters may not be achieved. On the other hand, if a too small $\rho$ is used, the algorithm attempts to impose a very tight control in the size of clusters and may fail to do so.

Let $\mathbf{w}_{\text {opt }}$ be the solution of the adaptive equaliser based on the criterion (19) that yields the correct signal constellation. Then the weight vectors which produce the same function value as $\bar{\eta}\left(\mathbf{w}_{\text {opt }}\right)$ are given by

$$
\mathbf{w}_{s}=\exp (j \phi) \mathbf{w}_{\mathrm{opt}}, \phi=0, \frac{\pi}{2}, \pi, \frac{3 \pi}{2} .
$$

Since the equaliser weights are adapted using a 4-cluster submodel at each sample via the gradient algorithm (21), the complexity is always compatible to the minimum complexity of the 4-QAM case, and is listed in Table I.

\section{Simulation STUDY}

Two performance criteria were used to assess the convergence rate of a blind equaliser. The first one was an estimated MSE at each adaptation sample based on a block of $N_{\mathrm{MSE}}$ data 


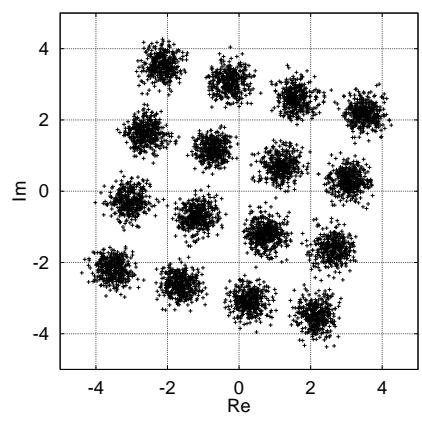

(a)

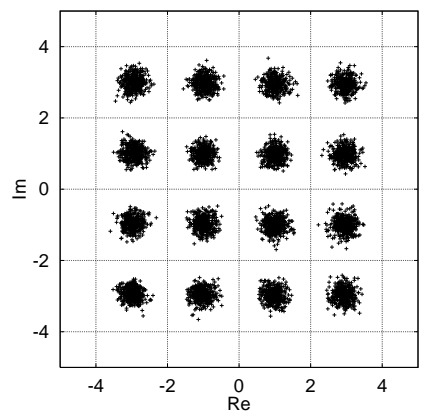

(b)

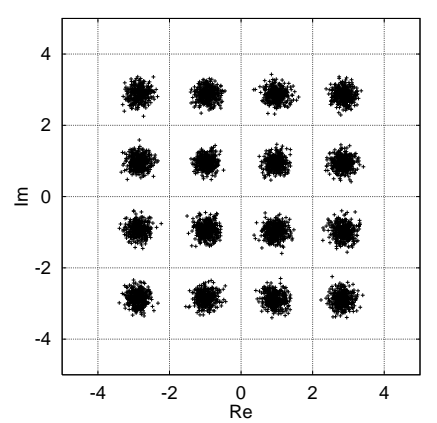

(c)

Fig. 2. Equaliser output signal constellations after convergence (a) the CMA, (b) the CMA+DD, and (c) bootstrap MAP.

samples. The second one was the maximum distortion (MD) measure defined by

$$
\mathrm{MD}=\frac{\sum_{i=0}^{n_{c}-1}\left|f_{i}\right|-\left|f_{i_{\max }}\right|}{\left|f_{i_{\max }}\right|}
$$

where $\left\{f_{i}\right\}_{i=0}^{n_{c}-1}$ was the combined impulse response of the channel and equaliser, $n_{c}=n+m-1$ was the length of the combined impulse response, and

$$
f_{i_{\max }}=\max \left\{f_{i}, 0 \leq i \leq n_{c}-1\right\} .
$$

The equaliser output signal constellation after convergence was also shown using $N_{\text {test }}=6000$ testing data samples.

The CIR, listed in Table II, was a 22-tap channel taken from [11] and the transmitted data symbols were 16-QAM. With a noise power of $\sigma_{e}^{2}=0.01$, the channel signal to noise ratio (SNR) was $27 \mathrm{~dB}$. The equaliser had 23 taps and the length of data samples for estimating the MSE was $N_{\mathrm{MSE}}=250$. The
CMA had to choose a small adaptive gain of $\mu=0.00001$ to avoid divergence, and the two adaptive gains of the concurrent CMA+DD equaliser were $\mu_{c}=0.00001$ and $\mu_{d}=0.0001$. For the bootstrap MAP equaliser, 2000 samples were used in the first stage with $\mu=0.002$ and $\rho=1.8$, while in the second stage the adaptive gain was $\mu=0.002$ with the cluster width $\rho=0.7$. The convergence performance of the three blind equalisers, in terms of the estimated MSE and MD measure, are depicted in Fig. 1. Both the concurrent CMA+DD and bootstrap MAP equalisers have similar steady state performance which are dramatical improvements over those of the CMA. For this example the bootstrap MAP equaliser has the fastest convergence speed. The three equaliser output signal constellations are shown in Fig. 2.

\section{CONCLUSIONS}

Two novel blind FIR equalisers for high-order QAM signalling, the concurrent CMA+DD and bootstrap MAP, have been compared, with the popular CMA as a benchmark. These two blind equalisers are attractive as they have low computational requirements that are only slightly more than the simple CMA. Simulation study has confirmed that these two blind equalisers outperform the CMA significantly. The results have also demonstrated that the bootstrap MAP equaliser has a faster convergence rate than the concurrent CMA+DD equaliser for the channel simulated.

\section{REFERENCES}

[1] Godard, D., Self-recovering equalization and carrier tracking in twodimensional data communication systems. IEEE Trans. Communications COM-28 (1980), 1867-1875.

[2] Treichler, J.R. and Agee, B.G., A new approach to multipath correction of constant modulus signals. IEEE Trans. Acoustics, Speech and Signal Processing ASSP-31 (1983), 459-472.

[3] Macchi, O. and Eweda, E., Convergence analysis of self-adaptive equalizers. IEEE Trans. Information Theory IT-30 (1984), 161-176.

[4] De Castro, F.C.C., De Castro, M.C.F. and Arantes, D.S., Concurrent blind deconvolution for channel equalization. In Proc. ICC'2001, Helsinki, Finland, June 11-15, 2001, Vol.2, pp.366-371.

[5] Benveniste, A. and Goursat, M., Blind equalizers. IEEE Trans. Communications COM-32 (1984), 871-883.

[6] Bellini, S. and Rocca, F., Blind deconvolution: polyspectra or Bussgang techniques? In Digital Communications, eds., E. Biglieri and G. Prati, North-Holland, Amsterdam, 1986, pp.251-263.

[7] Haykin, S., ed., Blind Deconvolution. Prentice Hall, Englewood Cliffs, NJ, 1994.

[8] Chen, S., McLaughlin, S., Grant, P.M. and Mulgrew, B., Reducedcomplexity multi-stage blind clustering equaliser. In Proc. ICC'93, Geneva, Switzerland, 1993, Vol.2, pp.1149-1153.

[9] Chen, S., McLaughlin, S., Grant, P.M. and Mulgrew, B., Multi-stage blind clustering equaliser. IEEE Trans. Communications 43 (1995), 701705 .

[10] Karaoguz, J. and Ardalan, S.H., A soft decision-directed blind equalization algorithm applied to equalization of mobile communication channels. In Proc. ICC'92, Chicago, U.S.A., 1992, Vol.3, pp.343.4.1343.4.5.

[11] Bateman, S.C. and Ameen, S.Y., Comparison of algorithms for use in adaptive adjustment of digital data receivers. IEE Proc. Pt.I 137 (1990), 85-96. 